\title{
Laser Ranging for Monitoring Water Waves in the new Deltares Delta Flume
}

\author{
M.Streicher ${ }^{\mathrm{a}}$, B. Hofland ${ }^{\mathrm{b}}$, R.C.Lindenbergh ${ }^{\mathrm{c}}$
}

${ }^{a}$ LWI Institute for Hydromechanics, TU Braunschweig, Germany -m.streicher@tu-bs.de

${ }^{\mathrm{b}}$ Deltares, Delft, The Netherlands - b.hofland@deltares.nl

${ }^{c}$ Dept.Of Geoscience and Remote Sensing, Delft University of Technology, The Netherlands r.c.lindenbergh@tudelft.nl

KEY WORDS: Laser, TLS, Delta Flume, Water Wave Measurements, Surface Correction

\begin{abstract}
:
Currently a new wave flume for hydraulic experiments near to prototype scale is under construction at Deltares. In the flume, modern measurement equipment will be installed. One of the potential sensor techniques is Terrestrial Laser Scanner (TLS) based range measurements of water waves. TLS range measurements are a very fast and accurate method for solid surfaces, providing temporal and spatial high resolution profile measurements. The conditions under which measurements of water surfaces with TLS are possible are however less well understood. The main objective of this work is to explore possibilities to apply TLS based range measurements of water waves in the new Delta Flume facility. Hence, influencing parameters on TLS based range measurements from water waves in the laboratory are identified from literature. The influencing parameters are further investigated in a test featuring the SICK LMS 511 and measuring the water surface in the $50 \mathrm{~m}$ TU Delft wave flume. Analysis of the results provides an insight into the possibilities and potential problems of the new measurement method. The obtained profile measurements from a originally straight still water surface showed a tendency to bend upwards at the side of the profile. For that reason a surface correction method based on the refraction of the laser beam when entering the water is elaborated. This method is part of the derived wave field reconstruction method. Finally, an optimized water wave measurement set-up for the new Delta Flume proposed.
\end{abstract}

\section{INTRODUCTION}

The need of protection against flooding is a crucial issue in the Netherlands and other low lying countries in the world. Floods may be induced by storm surges over the North Sea, which then result in both high water levels and large waves loading the coastal defense structures. To test the impact of waves on different coastal defense structures and materials, Deltares operates the Delta Flume facility, which is one of the largest wave flume facilities in the world (Figure 1). Currently a new Delta Flume (length $=230 \mathrm{~m}$, width $=5 \mathrm{~m}$, height $=9.5 \mathrm{~m}$ ) is constructed in Delft (Hofland et al. 2012). The flume is crucial for the Netherlands to model coastal structures and to assess their safety against major wave attack, storm surges and flooding.

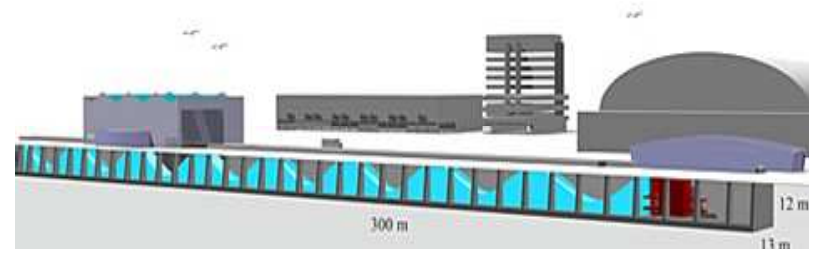

Figure 1: Design of the new Delta Flume facility to be opened in Nov. 2013

Typically, conventional capacitance and resistance type of wave probes (Whittenbury et al. 1959) are used to measure the water waves generated in the flume. They are considered an intrusive single point measurement.

It is the aim to equip the flume with the most advanced water sensor devices. One of the potential sensor techniques is TLS based range measurement of water waves. Compared to conventional laboratory wave measurement devices (e.g. resistance wave probes), TLS based range measurements are considered non-intrusive profile measurements. Non-intrusive means that there is no disturbance of the wave due to physical penetration of the water. Instead of a single point, a profile measurement is enabled, resulting in a temporally and spatially continuous signal of the water surface. Waves generated in laboratory wave flumes are expected to have the same shape in cross flume direction. Hence, a profile measurement in flume axis direction is sufficient. The measurements should enable the extraction of the wave parameters, height, length and period as well as the characterization of wave breaking and swash movements. The obtained profiles should also serve for the validation of numerical models.

In this study, the parameters that influence TLS based range measurements of water waves are investigated. Experiments with a SICK LMS 511 device are conducted in the small wave flume facility of the TU Delft. Processing of the data gives an insight into the possibilities and potential problems of the new measurement method. It reveals which steps of filtering, averaging and correction are necessary to reconstruct a wave field from the obtained data.

Finally, the knowledge gained is transferred to the Delta Flume facility and an optimized TLS based measurement set-up for the Delta Flume is proposed.

\section{OVERVIEW OF RELATED WORK}

TLS based range measurements of water waves are relatively new. Hence, parameters which influence the quality of the measurements are first identified in a parameter inventory. The classification is based on the categories elaborated by Soudarissanane et al. (2011) and adapted for the measurement of water waves. Relevant TLS, atmosphere/geometry, water and combined parameters, resulting from at least two of the above mentioned parameters, are given in Table 1. 


\begin{tabular}{|ll|}
\hline TLS & Parameter \\
& $\begin{array}{l}\text { Beam (pulsed or continuous, power, divergence, } \\
\text { diameter) } \\
\text { Resolution (spectral/wavelength, angular, range, } \\
\text { radiometric, temporal) } \\
\text { Opto-mechanical device (mirror) } \\
\text { Measurement principle (time-of-flight, phase- } \\
\text { shift, peak detection method) } \\
\text { Error (systematic, statistic, accuracy, precision) } \\
\text { PC/Software (threshold, filtering, interpolation) } \\
\text { Atmosphere/ } \\
\text { Lighting } \\
\text { Weather conditions (fog, cloud, rain, } \\
\text { temperature) } \\
\text { Set-up (height, distance, incidence angle) } \\
\text { Surface roughness (capillary waves, foam, } \\
\text { turbulence, ripples, wave breaking) } \\
\text { Turbidity (naturally, additives) } \\
\text { Wave geometry (height, period) } \\
\text { Wave celerity } \\
\text { Laser beam footprint } \\
\text { Laser beam point spacing } \\
\text { Grazing angle } \\
\text { Specular or diffuse reflection } \\
\text { Spike effects } \\
\text { Shadowing } \\
\text { Surface ambiguity } \\
\text { Parallel movement of TLS and wave }\end{array}$ \\
Combined
\end{tabular}

Table 1: Influencing parameters on scanning water waves

A distinction is made between airborne, outdoor terrestrial and laboratory laser range measurements of water surfaces. Airborne LiDAR (Light Detection And Ranging) measurements are mainly applied to topographic measurements of landscapes, bottom detection and bathymetry measurements (Guenther et al. 2000), but also for delineation and classification of the waterland boundary along rivers (Höfle et al. 2009). Several approaches to apply TLS based range measurements to water waves in the coastal zone are reported by Maslov et al. (2006) with the focus on the required laser power. Outdoor TLS based measurements of water waves are conducted by Harry et al. (2010), Vousdoukas et al. (2011) and Park et al. (2011). Blenkinsopp et al. (2010) used a SICK device in the coastal swash zone and reconstructed a continuous profile of the breaking wave and run-up tongue. Belmont et al. (2006) focused on the influence of the incidence angle on TLS based wave measurements in the coastal zone.

Only few applications of TLS devices applied in a laboratory wave flume are reported. Using the laser profiler SICK LMS 200 with $905 \mathrm{~nm}$ wavelength, Allis et al. (2011) found that water turbidities exceeding 40 NTU (Nephelometric Turbidity Units) and incidence angles below $55^{\circ}$ are required for reliable measurements. Kaolinit $\left(\rho=2.65 \mathrm{~g} / \mathrm{cm}^{3}, \quad \mathrm{D} 50=20 \mu \mathrm{m}\right)$ was used as an additive. The resulting RMSE between TLS and wave probe measurements is $3.62 \mathrm{~mm}$, with wave steepness between 0.05 and 0.2. Blenkinsopp et al. (2012) conducted experiments with the same device but focused on the recording of the wave shoaling process (increasing wave heights when entering shallow waters). In this more dynamic setting, a RMSE between TLS and wave probe measurements of $6.1 \mathrm{~mm}$ is reported with significant wave heights between $0.16 \mathrm{~m}$ and $0.3 \mathrm{~m}$.

\section{SMALL FLUME EXPERIMENT}

In this section, scan experiments performed in a small flume are analysed. First, the experimental set-up is discussed in section 3.1. Based on the findings from the parameter inventory (Section 2), the experimental set-up is divided in TLS, atmosphere/geometry and water parameters. Possibilities and problems when reconstructing a still water surface from the obtained signal are described in section 3.4. A detailed surface correction method will be elaborated in section 3.5.

\subsection{Wave Flume and Water Parameters}

The TU Delft Wave Flume facility is $50 \mathrm{~m}$ long, $0.8 \mathrm{~m}$ wide and $1.05 \mathrm{~m}$ high. During experiments, a water level of $0.6 \mathrm{~m}$ was present as shown in Figure 2. At the one end, a mechanical wave maker generated the required waves. On the other side, a dissipative slope was installed to make sure that disturbing reflection phenomena were minimized.

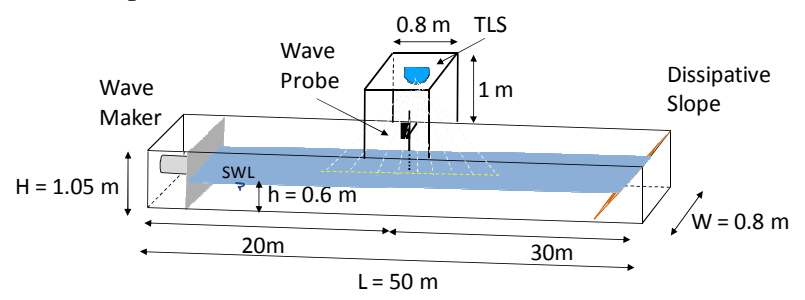

Figure 2. TU Delft wave flume

Approximately $20 \mathrm{~m}$ from the wave paddle, the TLS device was mounted. The turbidity of the water varied between 0 NTU and 90 NTU during experiments, using the additive Kaolinit $\left(\rho=2.65 \mathrm{~g} / \mathrm{cm}^{3}, \mathrm{D} 50=20 \mu \mathrm{m}\right)$. The Kaolinit was suspended in water in an extra basket and then manually poured into the flume over a total length of approximately $8 \mathrm{~m} \mathrm{(4} \mathrm{m} \mathrm{to} \mathrm{each}$ side of the TLS). Stirring with a stick should optimize the equal distribution of the Kaolinit along the flume axis. A probe was taken below the TLS device and the turbidity was measured.

Regular waves with wave heights $\mathrm{H}_{\mathrm{s}}$ between $0.05 \mathrm{~m}$ and $0.2 \mathrm{~m}$ were generated. Corresponding wave periods $\mathrm{T}_{\mathrm{p}}$ varied between $0.9 \mathrm{~s}$ and $1.7 \mathrm{~s}$.

In order to increase the turbulence in the wave field and to improve the reflectivity of the water, irregular waves that were occasionally breaking (whitecapping) were tested as well.

Before each test, a white piece of paper was put on the water surface in the area of the measured profile to give a first estimation of the 'true' water surface.

\subsection{Atmosphere and Geometry Parameters}

Since the experiments were conducted inside the TU Delft laboratory, artificial lighting from the ceiling was present. A wooden mounting construction for the TLS device was adjusted on the sidewalls of the wave flume (Figure 3 ).

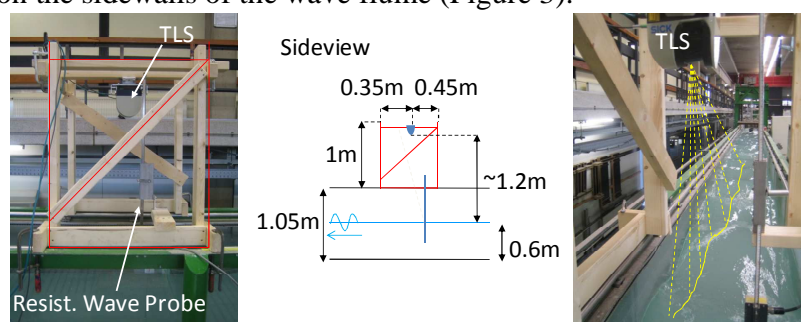

Figure 3. TLS mounting construction

The construction should allow for installation of the TLS device in sufficient height above the water and a free field of view on 
the water surface, without objects interrupting the laser beam. The final prototype had a height of $1 \mathrm{~m}$, a width in flume direction of $0.8 \mathrm{~m}$ and a depth perpendicular to the flume axis of $0.8 \mathrm{~m}$. Since the TLS device was mounted below the topmost wooden beam, the source of the laser beam is approximately $0.2 \mathrm{~m}$ lower than the height of the mounting construction. This leads to a vertical distance towards the still water surface of approximately $1.2 \mathrm{~m}$.

\subsection{TLS Parameters}

A SICK LMS511 Pro SR is used in the experiments (Figure 4). It is equipped with a pulsed laser beam and uses the Time-of-Flight (ToF) measurement principle. The laser light has a wavelength of $905 \mathrm{~nm}$. The beam diameter at the opening is $13.6 \mathrm{~mm}$ and the beam divergence is $11.9 \mathrm{mrad}$. Although it is a laser

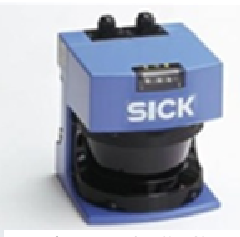

Figure 4. SICK

LMS511 Pro SR profiler (measurement in helical mode) the term TLS will be used in this thesis to describe the instrument. A scanning rate of $50 \mathrm{~Hz}$ and an angular resolution of $0.5^{\circ}$ are applied. The function to record multiple echoes is switched off, as well as the fog filter, to avoid a lower sensitivity in the near range $(<7 \mathrm{~m})$. The specification sheet gives a total systematic plus statistical error of $31 \mathrm{~mm}$ for ranges between $1 \mathrm{~m}$ and $10 \mathrm{~m}$. The laser power of the device is $15 \mathrm{mj} /$ pulse.

\subsection{Reconstructed Still Water Surface Profile}

The simple case of a still water surface measurement was used to identify several problems of this method. The results can later be adapted to dynamic water wave conditions.

Parts of the analysis require the conversion of the scan points from polar to Cartesian coordinates. Internal data collection of the TLS device is in polar coordinates.

Scan points taken from locations very remote from the expected water surface are excluded (e.g. points from the ceiling, the wave maker at the end of the flume or points from the bottom of the flume). A vertical buffer between $\mathrm{y}=1 \mathrm{~m} \div 1.2 \mathrm{~m}$ and $\mathrm{a}$ horizontal buffer of $\mathrm{x}=-6 \mathrm{~m} \div+6 \mathrm{~m}$ from the laser source were applied for this purpose.

The recorded echoes decrease for more gentle incidence angles. Due to the wave action, this also changes in time. To obtain a measurement where most of the area gives continuous results and to quantify this effect, a 95\% Field of View (FoV) region is calculated. The laser beam moves over the water surface in predefined steps and frequency. The percentage of validated echoes for each location over the whole test duration is determined.

The $95 \%$ FoV region is then defined as the distance between the minimum and maximum location with at least $95 \%$ recorded echoes, as shown in Figure 5. In nadir, single locations didn't show $95 \%$ recorded echoes, but points to the left and the

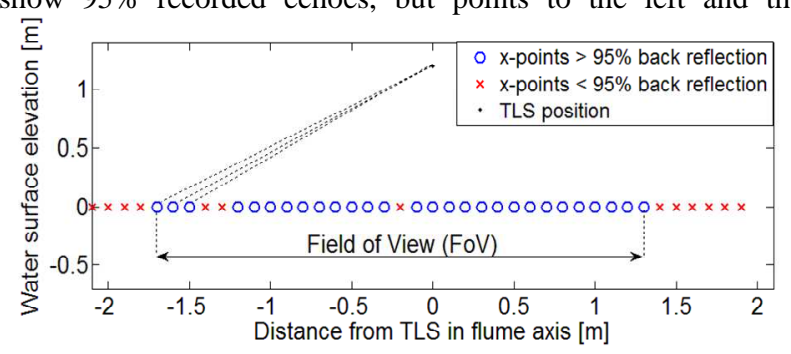

Figure 5. Definition 95\% Field of View (FoV) right did so. These missing points are simply interpolated using two neighboring points. The same was true for locations with less than $95 \%$ recorded echoes near the edge of the profile.

The point spacing on the water surface is irregular due to the combination of increasing ranges and higher incidence angles. To provide an equal distribution, the recorded echoes are interpolated in steps of $0.01 \mathrm{~m}$ over the whole profile. This is about equal to the densest point spacing around nadir. The signal was then temporally and spatially averaged.

\subsection{Surface Correction Method}

It was observed that the obtained still water surface profiles showed a tendency to bend upwards towards the side, although the original water surface is a horizontal line. It is assumed that the laser beam travels a certain distance B through the water column before being reflected or scattered by any particle (Tamari et al. 2011). Hence, the laser beam experiences refraction and a decrease in velocity when entering the water column. For the range calculation, the TLS however assumes a constant velocity of the laser beam and a measurement which is on a straight line (Figure 6).

Therefore, the obtained results seem to be more distant and of higher elevation than in reality. This could result in the upward bending towards the profile edges. To correct for this and to obtain the desired water surface coordinates, a surface correction method after Smith et al. (2011) is derived.

The refractive index of water with an incidence laser wave length of $905 \mathrm{~nm}$ is $\mathrm{n}_{\text {water }}=1.32699$ (Daimon \& Masumura 2007) and the refractive index of air is $n_{\text {air }}=1.00027$.

The horizontal and vertical distance towards the water surface $\Delta \mathrm{x}_{\mathrm{v}}$ and $\Delta \mathrm{y}_{\mathrm{v}}$ respectively are calculated from the virtually

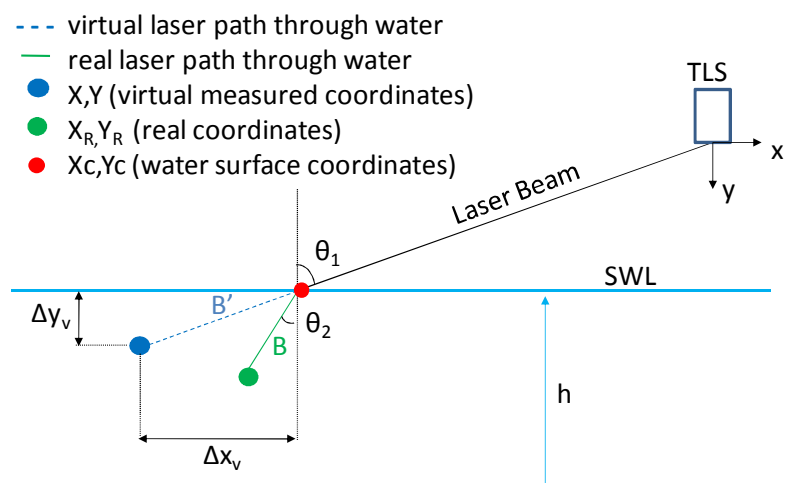

Figure 6. Surface correction after Smith et al. (2011)

obtained coordinates $(\mathrm{X}, \mathrm{Y})$. The TLS device assumes no refraction, so the angle in water is the same as the incidence angle. Furthermore, it assumes that the laser beam is traveling with the same velocity in water. Since in reality the laser beam is traveling slower in water than in the air, the length B must be multiplied with the quotient of the air- water refractive index to obtain B': 
Finally the water surface coordinates are calculated with:

$$
\begin{aligned}
& X_{c}=X-\Delta x_{v}=X-\sin \theta_{1} * B * \frac{n_{2}}{n_{1}} \\
& Y_{c}=Y-\Delta y_{v}=Y-\cos \theta_{1} * B * \frac{n_{2}}{n_{1}}
\end{aligned}
$$

Where $\quad$ B $=$ Dist. laser beam travels under water $[\mathrm{m}]$ $\theta_{1} \quad=$ Incidence angle $\left[{ }^{\circ}\right]$

$\mathrm{n}_{1} \quad=$ Refractive index air [-]

$\mathrm{n}_{2} \quad=$ Refractive index water [-]

$\mathrm{X}, \mathrm{Y}=$ Virtually measured coordinates $[\mathrm{m}]$

$\mathrm{X}_{\mathrm{c}}, \mathrm{Y}_{\mathrm{c}}=$ Water Surface coordinates $[\mathrm{m}]$.

The incidence angle is obtained from the TLS device. The question is how to determine the distance B. If indeed the upward bending of the sampled water profile can be explained by the slower velocity in water, the correction should result in an almost planar water surface profile. Thus, a backwards iterative approach to determine $\mathrm{B}$ is chosen by determining the value of $B$ that results in the most straight profile. It is assumed that for a given water turbidity B is constant for all incidence angles. The root mean square error of the vertical distance to the water surface $\Delta y_{v}$ in Equation (1) is calculated as a function of $\mathrm{B}$. Where $\mathrm{N}$ is the total number of measured points.

$$
\operatorname{Min}(R M S E)=\sqrt{\frac{\sum_{i=1}^{N}\left(\Delta \hat{y}_{v}(B)-\Delta y_{v i}(B)\right)^{2}}{N}}
$$

The $\Delta \mathrm{y}_{\mathrm{v}}$ resulting in the minimum RMSE (most planar surface) is calculated. The corresponding B is chosen for the surface correction (Equation 2). Figure 7 shows a water surface profile before (blue dots) and after surface correction (red dots).

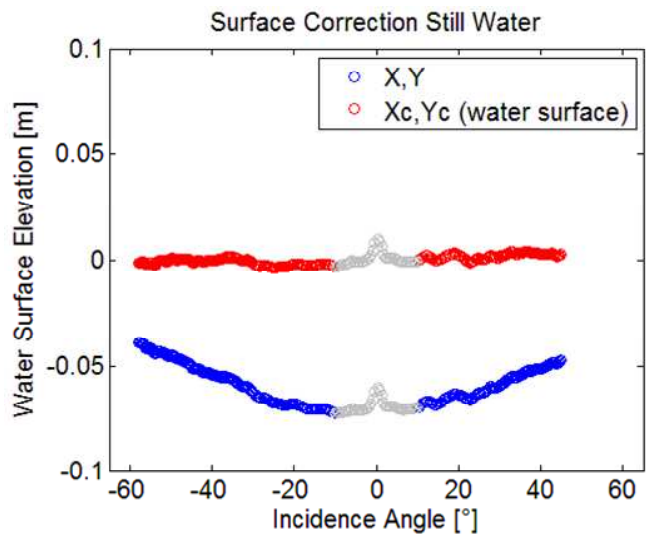

Figure 7: Still water surface profile before (blue), and after (red) surface correction

Only incidence angles between $10^{\circ}$ and $60^{\circ}$ are considered (In nadir, between $0^{\circ} \div 10^{\circ}$ and highlighted in grey, the signal deteriorates). The upward bending of the edges is corrected and a rather horizontal water surface profile is obtained.

Two problems are observed, which lead to difficulties when applying the surface correction method. In Figure 8, a sloped
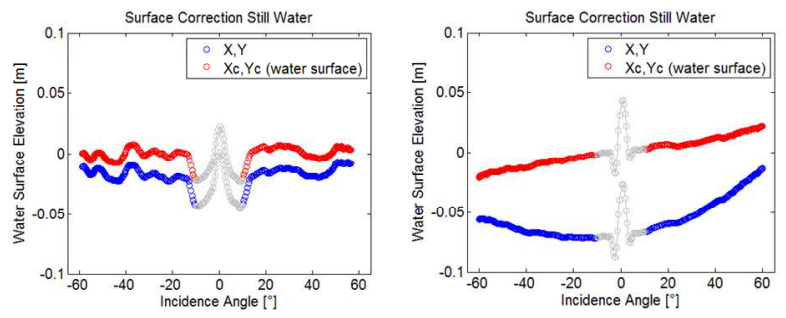

Figure 8: Unequally distributed Kaolinit (left) and aslope TLS installation (right)

water surface profile is displayed on the right graph. It's probably a systematic error due to the non-horizontal installation of the TLS device above the wave flume. Still, the corrected water surface profile is much straighter and the upward bending of the edges is corrected.

In Figure 8 on the left side, unequally distributed Kaolinit (varying turbidity in flume axis) is assumed to be the reason for the scatter in water surface elevation. In this case, surface correction is hardly possible.

A more sophisticated steering of the additive is demanded to guarantee an equal distribution of the turbidity along the flume axis.

Apart from this, the proposed surface correction method performs well in the way that the upward bending profile edges are corrected and a mostly planar water surface profile is obtained. It is assumed that the scatter in consecutive measurements and the distance $\mathrm{B}$, the laser beam travels through the water will decrease with increasing water turbidities.

However, the iteratively derived distance B, i.e. the distance the laser beam traveled under water, seems to be overestimated. A possible explanation is that the upwards bending profile edges are not only due to the refraction phenomena. Here, further research is required to quantify the specific influence of the refraction.

\section{PROPOSED WATER WAVE MEASUREMENT SET- UP FOR THE NEW DELTA FLUME}

It is the purpose of this section to propose an optimized set-up for TLS based range measurements of water waves in the new Delta Flume. Hence, optimized TLS, geometry/atmosphere and water parameters are determined and post processing steps are described.

\subsection{TLS Parameters}

The SICK LMS511 was found to give good results in the green water (defined as the water where waves are generally unbroken) of the small flume with ranges between $1.2 \mathrm{~m}$ and $4 \mathrm{~m}$. In Figure 9, a reconstructed wave field is displayed. The 95\% Field of View (FoV) is applied and the wave measurement is interpolated and spatially averaged with the 5th moving window. The surface correction method presented in section 3.5 is applied. At least one full wave length is visible. 
The question is how this same device will perform in the larger Delta Flume dimensions.
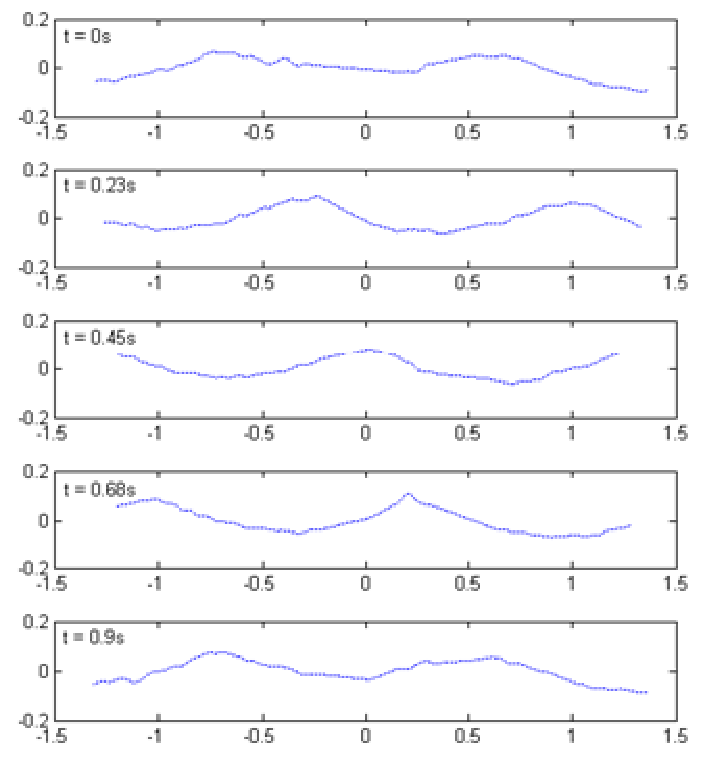

Figure 9: Wave measurement

Good results are reported for the use of the SICK device in the wave run-up zone of the old Delta Flume. There, a very foamy and turbulent environment provides better reflection characteristics. Up to distances of $20 \mathrm{~m}$, breaking waves were detected. When measuring a still water surface in the Delta Flume with ranges between $4 \mathrm{~m}$ and $8 \mathrm{~m}$, no good results were obtained.

The SICK LMS511 is equipped with a near infrared laser $(\lambda=905 \mathrm{~nm})$. The use of near infrared lasers for water surface detection is in line with the Optech Shoals approach for airborne LiDAR bathymetry measurements (Guenther et al. 2000).

The SICK LMS511 is provided with the pulsed Time-of-Flight (ToF) measurement principle. Reported TLS based range measurements from water waves are also obtained with ToF measurement devices. Guenther et al. (2000) observed, that during airborne LiDAR bathymetry measurements Raman reflection from the water is detected. It is not clear what happens to the phase-shift (PS) range measurements if a different wavelength (emitted by the water) than the incidence wavelength (emitted by the laser) is coming back to the device. Also, no results were obtained using a phase- shift (PS) device in the TU Delft wave flume (Faro Photon 120). Although there are more parameters influencing the quality of the measurements, it is assumed that the ToF range measurement principle is more robust for scanning water waves. However, further research is required to prove this assumption.

Water is a very low reflective surface. Therefore, intensity filters (fog filter etc.), frequency filters or comparable internal TLS thresholds should have the possibility to be switched off. In this way, as much data points as possible are obtained from the water surface. If required, filtering of the point cloud can be done manually in post processing (Section 3.4).

Very sensitive receivers with high radiometric resolution are expected to better measure the low intensity echoes from the water surface and the small changes in intensity.

\subsection{Geometry and Atmosphere Parameters}

Two different locations in the new Delta Flume are identified for the TLS measurements. The first location is placed in the so- called green water, defined as the water were waves are generally unbroken. Wave profiles and the accompanying wave parameters are measured in this location. Several proposed measurement locations for the green water location are given in Figure 10.

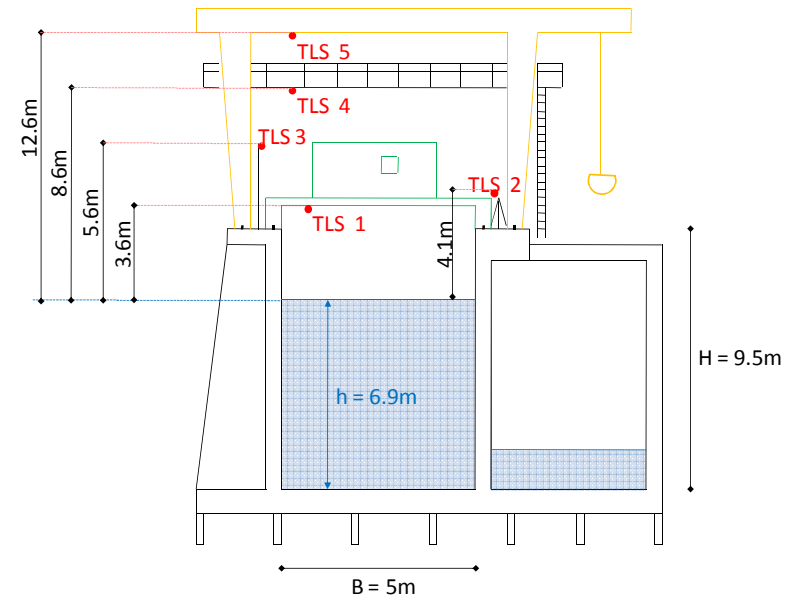

Figure 10: Possible TLS measurement locations in the new Delta Flume

The second location is placed in the wave run-up zone of the Delta Flume. The foamy and turbulent environment of the breaking waves will provide better reflections for the TLS device.

The measurement location along the flume axis should always be near the area of interest. That way errors due to high incidence angles, resulting in ellipsoidal footprints and signal deterioration (Sourarissanane et al. 2011), are reduced.

Due to easy access, flexibility in the flume axis and due to the largest distance to the water surface without interrupting objects, TLS4 (in Figure 10) is considered the most preferred measurement location. Each TLS location is slightly shifted from the middle of the flume to easily implement a small slant angle. A slant angle greater than $4^{\circ}$ prevents a saturated signal in nadir (Allis et al. 2011).

In the conducted experiments, no influence of illumination conditions on the measurements could be observed. This is in line with the observations by Irish et al. (2006).

It will be even more challenging to receive a reflection from the still water surface over increasing distances. The required laser power will increase with larger ranges, as present in the Delta Flume. The SICK LMS511 provided good results in the small scale wave flume with ranges between $1 \mathrm{~m} \div 3 \mathrm{~m}$ and a laser power of $15 \mathrm{~mJ} / \mathrm{pulse}$. Hence, the estimated required laser power, based on the laser range equation and power of the SICK LMS 511 device, is $0.77 \mathrm{~J} /$ pulse for measurement location TLS4.

\subsection{Water Parameters}

Different additives were used during the experiments to guarantee for the required turbidity of the water. Mainly, the additive Kaolinit $\left(\rho=2.65 \mathrm{~g} / \mathrm{cm}^{3}, \mathrm{D} 50=20 \mu \mathrm{m}\right)$ was used. As an experiment, also Magnesium powder ("Moon Dust", $\rho=1.738 \mathrm{~g} / \mathrm{cm}^{3}$ ) was tested. Since it has a lower density than Kaolinit, it sank slower, allowing for a longer measurement time. It was difficult to dissolve the magnesium powder in water due to the fact that it was sticking together in larger clumps. Accordingly, Kaolinit is the preferred additive for the time being.

The measurements in the green water with unbroken waves will be comparably difficult to obtain. Here, the turbidity must 
exceed 40 NTU (Allis et al. 2011) to enable TLS based range measurements from the water surface. Advanced turbidity steering is required to guarantee for equally distributed Kaolinit along the flume axis. Measurements in the wave run-up zone are simplified by the higher turbulence in the water which forces the particles in suspension. Also, the foamy environment due to wave breaking processes increases the reflectivity.

\subsection{Post Processing}

Basically, the conversion, filtering, interpolation, averaging and correction steps presented in section 3.4 should be adapted to wave conditions. Spectral filtering is recommended and the surface correction method (section 3.5) can be used for a changing wave face as well.

\section{CONCLUSIONS}

The applicability of a TLS based range measurement of water waves in the Delta Flume has been examined. A ready to use measurement set- up has not been applied yet. Questions about the physical processes in laser- water interaction remain. Nevertheless, some important steps to reach this ultimate goal are presented in this work.

A parameter inventory was carried out, identifying and summarizing the influencing parameters on TLS based range measurement of water waves.

A SICK LMS511 device was applied in the small scale wave flume facility of the TU Delft. It was the purpose to implement a working measurement set-up, which can later be adapted to a measurement set-up in the large scale Delta Flume. A water wave profile extending one wavelength was visible in the reconstructed wave signal.

When reconstructing the wave field, several issues are observed (e.g. the peak in nadir, upwards bending of the profile edges, scatter and bias in the measurements) and analyzed for still water conditions. Methods to finally reconstruct a wave field from the signal were described (coordinate transformation, geometrical filter, $95 \% \mathrm{FoV}$, interpolation and averaging). To correct for the upward bending of the profile edges, a surface correction method was elaborated. Based on the results, an optimized measurement set-up for the Delta Flume is proposed.

\section{REFERENCES}

Allis, M.J., Peirson, W.L., Banner, M.1., 2011. Application of LIDAR as a Measurement Tool for Waves. Proceedings of 21 st International Offshore and Polar Engineering Conference, Maui, Hawaii USA, pp. 373-379.

Belmont, M.R., Horwood, J.M.K., Thurley, R.W.F., Baker, J., 2006. Shallow Angle Wave Profiling LIDAR. Journal of Atmospheric and Oceanic Technology, 24, pp. 1150-1156.

Blenkinsopp, C.E., Mole, M.A., Turner, I.L., Peirson, W.L., 2010. Measurements of the Time- Varying Free- Surface Profile Across the Swash Zone Obtained Using an Industrial LIDAR. Coastal Engineering, 57, pp. 1059-1065.

Blenkinsopp, C.E., Turner, I.L., Allis, M.J., Peirson, W.L., Garde, L.E., 2012. Application of LiDAR technology for Measurement of Time- Varying Free- Surface Profiles in Laboratory Wave Flume. Coastal Engineering, 68, pp.1-5.

Daimon, M., Masumura, A., 2007. Measurement of the refractive index of distilled water from the near- infrared region to the ultraviolet region. Applied Optics, 46, pp. 3811-3820.
Guenther, G.C., Cunningham, A.G. LaRocque, P.e., Reid, D.J., 2000. Meeting the Accuracy Challenge in Airborne Lidar Bathymetry. Proceedings of EARSeL-SIG-Workshop LiDAR, Dresden, Germany, pp. 1-21.

Harry, M., Zhang, H., Lemckert, C., Colleter, G., 2010. 3D Spatial Definition of a Water Surface. Proceedings of the 9th ISOPE Pacific/Asia Offshore Mechanics Symposium, Busan, Korea, pp. 255-261.

Hofland, B., Hoffman, R., Lindenbergh, R., 2012. Wave Measurement Techniques for the new large-scale Delta Flume Proceedings of 4th International Conference on the Application of Physical Modelling to Port and Coastal Protection - Coastlab12, Ghent, Belgium.

Höfle, B., Vetter, M., Pfeifer, N., Mandlburger, G., Stötter, J., 2009. Water Surface Mapping From Airborne Laser Scanning Using Signal Intensity and Elevation Data. Earth Surface Processes and Landforms, 34, pp.1635-1649.

Irish, J.L., Wozencraft, J.M., Cunningham, A.G., Giroud, C., 2006. Nonintrusive Measurement of Ocean Waves: Lidar Wave Gauge. Journal of Atmospheric and Oceanic Technology, 23, pp. 1559- 1572 .

Maslov, D.V., Fadeev, V.V., Lyashenko, A.I., 2000. A ShoreBased LIDAR for Coastal Seawater Monitoring. Proceedings of EARSeL-SIG-Workshop LiDAR, Dresden, Germany, pp. 46-52.

Park, H.S., Sim, J.S., Yoo, J.; Lee, D.Y., 2011. Breaking Wave Measurement Using Terrestrial LIDAR: Validation With Field Experiment on the Mallipo Beach. Journal of Coastal Research, 64, pp.1718-1721.

Soudarissanane S., Lindenbergh R., Menenti M., Teunissen P., 2011. Scanning Geometry: influencing factor on the quality of Terrestrial Laser Scanning points. ISPRS Journal of Photogrammetry and Remote Sensing, 66(4), pp. 389-399.

Tamari S., Mory J., Guerrero-Meza V., 2011. Testing a nearinfrared Lidar mounted with a large incidence angle to monitor the water level of turbid reservoirs. ISPRS Journal of Photogrammetry and Remote Sensing, 66, pp. 85-91.

Smith M., Vericat D., Gibbins C., 2012. Through-water terrestrial laser scanning of gravel beds at the patch scale. Earth Surface Processes and Landforms, 37, pp.411-421.

Vousdoukas, M.I., Wübbold, F., Hentschel, M., Wagner, B., 2011. Towards Autonomous Coastal Monitoring Using 3D Laser Range Scanners and Camera Vision.

Whittenbury, C.G., Huber, E.A., Newell, G.S. 1959. Instrument for Measuring Water Waves. The Review of Scientific Instruments, 30, pp. 674-676.

\section{ACKNOWLEDGEMENT}

We would like to thank the Department of Hydraulic Engineering at TU Delft for providing the wave flume facility and SICK company for providing the TLS device. Thanks to Nico Valentini who successfully continued this work. The new Delta Flume and its instrumentation are funded by the Dutch Government via the Fonds Economische Structuurversterking. 